\title{
FGF21 promotes wound healing of rat brain microvascular endothelial cells through facilitating TNF-a-mediated VEGFA and ERK1/2 signaling pathway
}

\author{
Weiting Chen ${ }^{1, A, D, F}$, Zhongen Shen ${ }^{2, A, B, E}$, Shuiqi Cai ${ }^{1, B, C}$, Long Chen ${ }^{1, C}$, Dabin Wang ${ }^{1, E}$ \\ ${ }^{1}$ No. 1 Department of Orthopedics, The Third People's Hospital, Cixi, China \\ ${ }^{2}$ Department of Anesthesiology, Cixi People Hospital, China \\ A - research concept and design; $\mathrm{B}$ - collection and/or assembly of data; $\mathrm{C}$ - data analysis and interpretation; \\ $D$ - writing the article; $E$ - critical revision of the article; $F$ - final approval of the article
}

Address for correspondence

\section{Dabin Wang}

E-mail: juf56689@126.com

Funding sources

None declared

Conflict of interest

None declared

Received on 0ctober 17, 2020

Reviewed on November 4, 2020

Accepted on February 19, 2021

Published online on June 11, 2021

Cite as

Chen W, Shen Z, Cai S, Chen L, Wang D. FGF21 promotes wound healing of rat brain microvascular endothelial cells through facilitating TNF-a-mediated VEGFA and ERK1/2 signaling pathway. Adv Clin Exp Med. 2021;30(7):711-720. doi:10.17219/acem/133494

DOI

10.17219/acem/133494

\section{Copyright}

Copyright by Author(s)

This is an article distributed under the terms of the

Creative Commons Attribution 3.0 Unported (CC BY 3.0)

(https://creativecommons.org/licenses/by/3.0/)

\begin{abstract}
Background. Wound healing is an essential physiological process in recovery after microsurgery.

Objectives. To further understand the functions of fibroblast growth factor 21 (FGF21), the roles of this factor were examined and its correlations with inflammation, vascular endothelial growth factor A (VEGFA) and ERK1/2 signaling pathway activation were analyzed.
\end{abstract}

Materials and methods. Rat brain microvascular endothelial cells (RBMECS) were treated with interleukin (IL)-1 13 and used for the experiments. Cell Counting Kit-8 (CCK-8) was used to detect the cell viability of RBMECs after treatment with LL-1B (1 ng/mL) and FGF21 or VEGFA overexpression, while changes in apoptosis were measured through flow cytometry. Migration was checked through the scratch test. FGF21 and VEGFA RNA expression was assessed using reverse-transcription quantitative polymerase chain reaction (RT-qPCR), which was also used to examine RNA expression of BCl-2, Bax and caspase-3. After IL-1B treatment and FGF21 overexpression, tumor necrosis factor alpha (TNF-a) and tumor growth factor $\beta 1$ (TGF- $\beta 1$ ) proteins level were observed with enzyme-linked immunosorbent assay (ELISA), which was also applied to check the expression of ERK1/2 after overexpression of FGF21 and VEGFA. PD98059 (50 $\mu M)$, an ERK1/2 inhibitor, was used to examine the roles of ERK1/2 in regulating cell viability and apoptosis.

Results. The IL-1 1 treatment significantly decreased the viability of RBMECS and TGF- $\beta 1$, but promoted cell apoptosis and TNF-a expression. FGF21 was downregulated by IL-1 3 treatment but its overexpression enhanced the viability of RBMECS and TGF- $\beta 1$ and ERK1/2 protein levels, and attenuated cell apoptosis and TNF-a. Upregulated TNF-a restrained cell viability and apoptosis of RBMECS after FGF21 overexpression, and its upregulation not only suppressed FGF21, but also VEGFA. Moreover, VEGFA suppression by TNF-a increased cell viability and ERK1/2 protein levels, and suppressed the apoptosis of RBMECs through its upregulation. However, PD98059 obstructed the functions of FGF21 and VEGFA.

Conclusions. FGF21 promoted the cell viability of RBMECs through upregulating TNF-a-mediated VEGFA and ERK1/2 signaling.

Key words: VEGFA, FGF21, ERK1/2 signaling pathway, RBMEC 


\section{Background}

Acute stroke remains the leading cause of morbidity and mortality worldwide, ${ }^{1}$ and is the main cause of disability. ${ }^{2}$ Stroke results from transient or permanent cerebral ischemia caused by arterial stenosis or occlusion, and common manifestations of this disease are focal neurological deficits. ${ }^{3,4}$ Intravenous thrombolysis and endovascular thrombectomy (EVT) are the 2 main treatments for revascularization. ${ }^{1}$ Recent randomized clinical trials have shown that EVT has a strong therapeutic effect on acute ischemic stroke caused by large vessel occlusions. ${ }^{5}$ However, damage to pig extracranial arteries, particularly endothelial cells, has been reported following EVT. ${ }^{6}$ A key step during wound healing after surgery is the transition from an inflammatory stage to a proliferative stage, where inflammation can recruit the innate immune system and help to remove dead tissues. ${ }^{7}$ However, prolonged inflammation can lead to uncontrolled activation, resulting in the inhibition of wound healing. ${ }^{8}$ Therefore, cytokines that can attenuate the influence of EVT on cerebral vessels and control the inflammatory response attract attention.

Fibroblast growth factor 21 (FGF21) belongs to the FGF19 subfamily of FGF growth factors, which have a low affinity for heparin and the ability to circulate throughout the body as endocrine factors binding at FGF receptors. ${ }^{9,10}$ It functions as a potent regulator of lipid or energy metabolism, and can lengthen the lifespan of mice with overexpression. ${ }^{11}$ Moreover, FGF21 is highly expressed in a mouse model of steatohepatitis, and injection of FGF21 attenuates the development of steatohepatitis in methionine- and choline-deficient mice. ${ }^{12}$ A study examining the effects of FGF21 on the progression of atherosclerosis has also reported that this growth factor can significantly downregulate the expression of inflammatory factors, including interleukin (IL)-1 $\alpha$, IL- 6 , and tumor necrosis factor alpha (TNF- $\alpha$ ), and can suppress the NF- $\mathrm{kB}$ signaling pathway in macrophages. ${ }^{13}$ In addition to repressing the inflammatory response, FGF21 has also been shown to facilitate wound healing in diabetic mice by promoting granulation, collagen deposition and re-epithelialization. ${ }^{14}$ Unlike other members in FGF family, FGF21 is the only one that has no mitogen activity and does not accelerate cancer occurrence. ${ }^{15}$ Thus, FGF21 has a great potential for clinical usage.

Following brain injury in rats, FGF21 can exert a protective effect by promoting neuronal survival, inhibiting the apoptosis of neurons through the PI3K/AKT signaling pathway, and decreasing cerebral infarct volume. ${ }^{16}$ As for angiogenesis in the brain, FGF21 has been shown to improve this process and to promote the healing of human brain microvascular endothelial cells through the formation of a FGF21/FGFR1/b-klotho complex and peroxisome proliferator-activated receptor gamma (PPAR $\gamma$ ) activation. However, whether FGF21 can mediate wound healing of the brain microvasculature in other ways is unknown.
Thus, we explored additional mechanisms whereby FGF21 could regulate angiogenesis and the healing of rat brain microvascular endothelial cells (RBMECs).

Angiogenesis is an important part of wound healing and vascular endothelial growth factor A (VEGFA) is one of the most efficient factors that promotes this process. ${ }^{17}$ In diabetic mice, the upregulation of TNF- $\alpha$ reduces angiogenesis through inhibiting VEGFA expression. ${ }^{18}$ In addition, the downregulation of VEGFA expression reduces the proliferation of endothelial cells, angiogenesis and re-epithelialization in mice. ${ }^{19}$ In human dermal fibroblasts, VEGFA can be induced by visfatin to promote cell proliferation and metastasis, and VEGFA upregulation in wound healing is associated with activation of the ERK pathway. ${ }^{20,21}$ Moreover, FGF21 has been reported to suppress melanogenesis in alpaca melanocytes by upregulating the expression of p-ERK1/2. ${ }^{22}$ Based on these findings, we hypothesized that FGF21 regulates VEGFA during wound healing and angiogenesis through activation of the ERK1/2 signaling pathway and regulation of the inflammatory response.

\section{Objectives}

Thus, to further understand the functions of FGF21, the roles of this factor were examined and its correlations with inflammation, VEGFA and ERK1/2 signaling pathway activation were analyzed.

\section{Materials and methods}

\section{Cell culture}

The RBMECs were purchased from Procell (Wuhan, China). These cells, which were isolated from brain tissues, are the main component of the blood-brain barrier. After the RBMECs were thawed in warm water at $37^{\circ} \mathrm{C}$, they were cultured in high-glucose Dulbecco's modified Eagle's medium (DMEM; Gibco, Waltham, USA) containing $10 \%$ fetal bovine serum (FBS), $100 \mathrm{U} / \mathrm{mL}$ of penicillin and $100 \mathrm{mg} / \mathrm{mL}$ of streptomycin (Gibco) at $37^{\circ} \mathrm{C}$ in $5 \% \mathrm{CO}_{2}$. The medium was replaced every 2 days and the cells selected for the experiments were all in the $3^{\text {rd }}$ to $6^{\text {th }}$ generation. To simulate an inflammatory environment, RBMECs were treated with rat IL-1 $\beta$ ( $1 \mathrm{ng} / \mathrm{mL}$; Sigma-Aldrich, St. Louis, USA) for $3 \mathrm{~h}$. After IL-1 $\beta$ treatment, the RBMECs were treated with the ERK1/2 specific upstream inhibitor PD98059 (50 $\mu \mathrm{M}$; MedChemExpress, Monmouth Junction, USA) for $1 \mathrm{~h}$. PD98059 is an effective inhibitor of the MEK signaling pathway through its blockade of MEK1 and MEK2 with an $\mathrm{IC}_{50}$ of $5 \mu \mathrm{M}$. It also inhibits the ERK1/2 signaling pathway by blocking phosphorylation. In preparation for the experiments, the cells were grouped and named the negative control (NC) group, the IL-1 $\beta$ group and the PD98059 group. 


\section{Cell transfection}

To analyze the functions of FGF21, VEGFA and TNF- $\alpha$, overexpression of these 3 genes was carried out. To create overexpressed FGF21, VEGFA and TNF- $\alpha$ in RBMECs, the pcDNA 3.1 vector (Invitrogen, Carlsbad, USA) was selected for transfection. Briefly, a compounded fragment of FGF21 was inserted into the pcDNA3.1 to create overexpressed FGF21. Overexpressed VEGFA and TNF- $\alpha$ were created in the same way. For cell transfection, RBMECs were seeded into 24 -well plates and cultured at $37^{\circ} \mathrm{C}$ with $5 \% \mathrm{CO}_{2}$. Transfection was performed until $50 \%$ confluence and Lipofectamine 3000 (Invitrogen) was used to mediate transfection. Then, $2 \mu \mathrm{L}$ of Lipofectamine 3000 and $0.5 \mu \mathrm{g}$ of RNA were added into serum-free Opti-MEM medium to incubate cells at $37^{\circ} \mathrm{C}$ for $2 \mathrm{~h}$. Later, the medium used for cell incubation was added for cell culturing at $37^{\circ} \mathrm{C}$ for $24 \mathrm{~h}$. The RNA expression of FGF21 and VEGFA was measured using reverse-transcription quantitative polymerase chain reaction (RT-qPCR), and TNF- $\alpha$ protein expression was measured using enzyme-linked immunosorbent assay (ELISA). After transfection, the cells were divided into the following groups: oeNC, oeFGF21, TNF- $\alpha$, oeVEGFA, oeFGF21 with TNF- $\alpha$, oeFGF21 with PD98059, and oeVEGFA with PD98059.

\section{RT-qPCR}

To measure the RNA levels of FGF21, TNF- $\alpha$ and VEGFA, total RNA was extracted using the TRIzol reagent (Invitrogen) from untreated and IL-1 $\beta$-treated RBMECs, according to manufacturer's instructions. Thereafter, reverse transcription was performed using a High-Capacity cDNA Reverse Transcription kit (Applied Biosystems, Foster City, USA). Based on the manufacturer's instructions, the PCR reactions were conducted using the 7500 Fast Real-Time PCR system (Applied Biosystems), and data were quantified using the $2^{-\Delta \Delta C t}$ method. The primers used are listed in Table 1.

The conditions of the PCR were predenaturation at $95^{\circ} \mathrm{C}$ for $1 \mathrm{~min}$ followed by 40 cycles of denaturation at $95^{\circ} \mathrm{C}$ for $30 \mathrm{~s}$, annealing at $60^{\circ} \mathrm{C}$ for $30 \mathrm{~s}$, and extension at $72^{\circ} \mathrm{C}$ for $30 \mathrm{~s}$. The results were obtained from three independent trials.

\section{CCK-8 assay}

Untreated and IL-1 $\beta$-treated RBMECs were seeded into 96-well plates at a density of $5 \times 10^{3}$ cells/well and incubated for $24 \mathrm{~h}$ at $37^{\circ} \mathrm{C}$ with $5 \% \mathrm{CO}_{2}$. Thereafter, $10 \mu \mathrm{L}$ of Cell Counting Kit-8 (CCK-8; Beyotime, Shanghai, China) was added to cells at $24 \mathrm{~h}, 48 \mathrm{~h}$ and $72 \mathrm{~h}$. Following this, the cells were cultured for $1 \mathrm{~h}$ and optical density (OD) values were detected at the $450 \mathrm{~nm}$ wavelength using a Varioskan ${ }^{\mathrm{TM}}$ LUX Multimode Microplate Reader (Thermo Fisher Scientific, Waltham, USA). All samples were run in a triplicate.

\section{Flow cytometry}

The RBMECs with and without IL- $1 \beta$ treatment were digested using $0.25 \%$ trypsin (Gibco) and rinsed twice in phosphate-buffered saline (PBS). Next, an Annexin V-FITC Apoptosis Detection kit (Beyotime) was used for apoptosis detection. Cells were resuspended at a density of $1 \times 10^{5}$ in $195 \mu \mathrm{L}$ of Annexin V-FITC binding buffer. Later, $5 \mu \mathrm{L}$ of Annexin V-FITC and $10 \mu \mathrm{L}$ of propidium iodide (PI) were added and the cells were cultured in darkness at $25^{\circ} \mathrm{C}$ for $20 \mathrm{~min}$. Thereafter, an Attune NxT Flow Cytometer (Invitrogen) was used to analyze the apoptosis rate of RBMECs. All measurements were repeated 3 times.

\section{Scratch test}

The scratch test was used to evaluate the migration ability of RBMECs. Briefly, untreated and IL-1 $\beta$-treated RBMECs were seeded into six-well plates and incubated until cells covered the plate. Thereafter, $20-\mu \mathrm{L}$ tips were used to create vertical scratches on the RBMECs. Later, the cells were rinsed with PBS 3 times to remove the scraped cells. The RBMECs were then cultured in serum-free medium at $37^{\circ} \mathrm{C}$ with $5 \% \mathrm{CO}_{2}$. Images were taken $24 \mathrm{~h}$ after incubation. All measurements were repeated 3 times.

\section{ELISA}

To examine the protein expression of TNF- $\alpha$, TGF- $\beta 1$ and ERK1/2, Rat TNF alpha ELISA (ab46070; Abcam, Cambridge, UK), Rat TGF- $\beta 1$ ELISA (ab119558; Abcam)

Table 1. Primer sequences

\begin{tabular}{|c|c|c|c|}
\hline RNA names & Forward & Reverse & Reference \\
\hline FGF21 & 5'-GGGTCAAGTCCGACAGAGGTAT-3' & 5'-ATCAAAGTGAGGCGATCCATAGA-3' & 23 \\
\hline VEGFA & 5'-CCAGGAGTACCCCG ATGAGATAG-3' & 5'-CTGGCTTTGGTGAGGTTTGATC-3' & 24 \\
\hline $\mathrm{BCl}-2$ & 5'-GAGTACCTGAACCGGCATCT-3 & 5'-GAAATCAAACAGAGGTCGCA-3' & 25 \\
\hline Bax & 5'-TTGCTACAGGGTTTCATCCA-3' & 5'-GAGTACCTGAACCGGCATCT-3' & 25 \\
\hline Caspase-3 & 5'-GGACCTGTGGACCTGAAAAA -3' & 5'-GCATGCCATAT CATCGTCAG-3' & 26 \\
\hline TNF-a & 5'-TACTGAACTTCGGGGTGATTGGTCC-3' & 5'-CAGCCTTGTCCCTTGAAGAGAACC-3' & 27 \\
\hline GAPDH & 5'-TGCCACTCAGAAG ACTGTGG-3' & 5'-GGATGCAGGGATGATGTTCT -3' & 25 \\
\hline
\end{tabular}


and ERK1 (pT202/pY204; Abcam) + ERK2 (pT185/pY187; Abcam) + total ERK1/2 ELISA (ab126445; Abcam) kits were used. According to the manufacturer's instructions, antibodies were first settled into 96-well plates and proteins from the RBMECs were then added. Next, biotinylated anti-TNF- $\alpha$, biotin-conjugated anti-rat TGF- $\beta 1$ monoclonal antibody, detection antibody Erk1 (T202/Y204)/ Erk2 (T185/Y187) and detection antibody Erk1/2 were mixed and incubated at room temperature. Thereafter, streptavidin-horseradish peroxidase (HRP) was pipetted into wells followed by washing with wash buffer. Next, TMB Substrate Reagent (BD Biosciences, Shanghai, China) was added and Stop Solution (BD Biosciences) was used for reaction termination. Color intensity was measured at 450 nm using a Varioskan ${ }^{\mathrm{TM}}$ LUX Multimode Microplate Reader (Thermo Fisher Scientific). The results for this experiment were from 3 independent trials.

\section{Statistical analyses}

Data are shown as mean \pm standard deviation (SD) and the results were analyzed using GraphPad Prism v. 7.0
(GraphPad Software, San Diego, USA) and SPSS v. 19.0 software (IBM Corp., Armonk, USA). Comparisons between the 2 groups were analyzed using the Student's t-test and multiple comparisons between the groups were performed using the S-N-K method. An alpha level of $\mathrm{p}<0.05$ was considered statistically significant.

\section{Results}

\section{IL-1 $\beta$ treatment induces cell apoptosis and inflammation of RBMECs, and decreases cell viability}

To confirm the effects of IL-1 $\beta$ treatment, the cell viability of RBMECs was first examined. The CCK- 8 assay showed that the viability of RBMECs was significantly decreased by IL-1 $\beta$ treatment as compared to untreated RBMECs ( $\mathrm{p}<0.05$, Fig. 1A). In addition, IL-1 $\beta$-treated RBMECs showed a significantly higher level of apoptosis rate compared to untreated RBMECs ( $<<0.05$, Fig. 1B). The RT-qPCR also indicated that RBMECs treated with
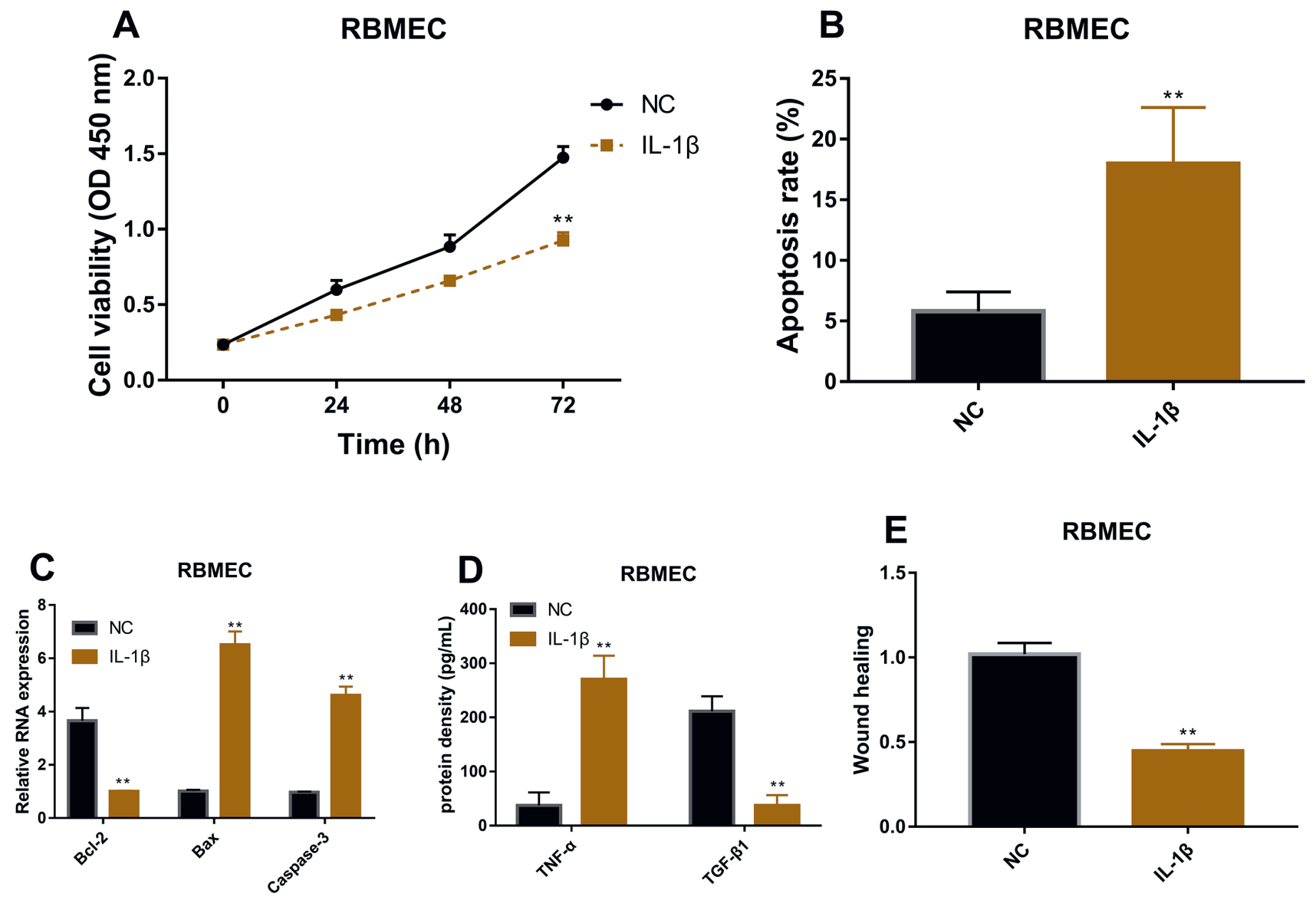

Fig. 1. IL-1 $\beta$ treatment induces cell apoptosis and inflammation of RBMECs, and decreases cell viability

A. Cell viability of untreated and IL-1 $\beta$-treated $(1 \mathrm{ng} / \mathrm{mL})$ RBMECs as detected using CCK- 8 (**p $<0.05$ compared to the NC group); B. Flow cytometry was used to examine apoptosis of untreated and IL-1 $\beta$-treated $(1 \mathrm{ng} / \mathrm{mL}$ ) RBMECs (**p $<0.05$ compared to the NC group); C. RNA expression of BCl-2, Bax and caspase-3 were evaluated with RT-qPCR in untreated and IL-1 $\beta$-treated ( $1 \mathrm{ng} / \mathrm{mL})$ RBMECs (**p $<0.05$ compared with the NC group); D. TNF- $a$ and TGF- $\beta 1$ protein levels in untreated and IL-1 1 -treated $(1 \mathrm{ng} / \mathrm{mL}$ ) RBMECs were measured with ELISA (** $\mathrm{p}<0.05$ compared to the NC group); E. Wound healing of untreated and IL-1 $\beta$-treated ( $1 \mathrm{ng} / \mathrm{mL}$ ) RBMECs was analyzed with the scratch test $\left({ }^{* *} \mathrm{p}<0.05\right.$ compared to the NC group). 

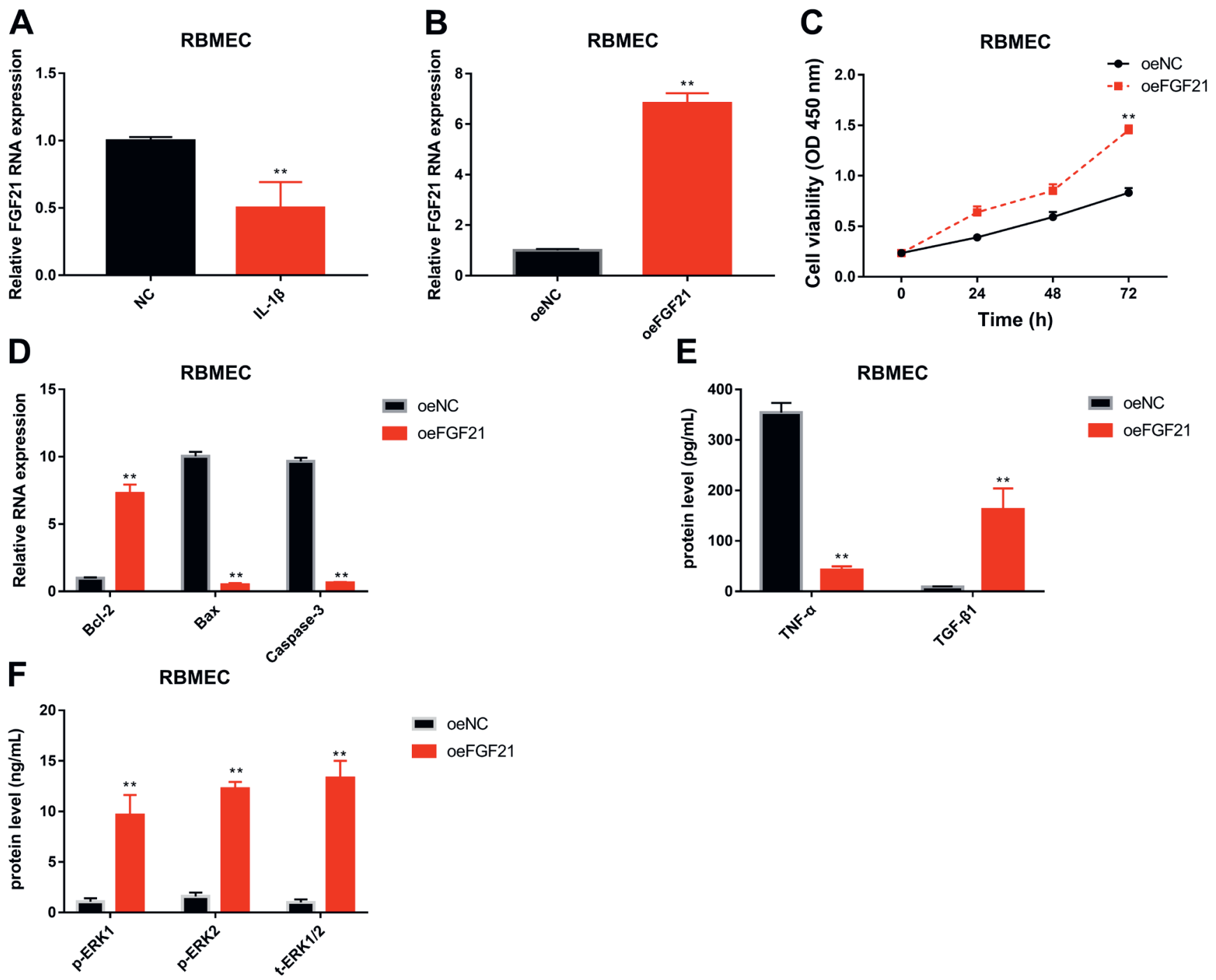

Fig. 2. Overexpression of FGF21 promotes cell viability, and inhibits cell apoptosis and inflammation of RBMECs via the ERK1/2 signaling pathway

A. FGF21 RNA expression in untreated and IL-1 1 -treated (1 ng/mL) RBMECs were measured using RT-qPCR $(* * 0<0.05$ compared to the NC group); B. RT-qPCR was used to analyze FGF21 RNA expression in RBMECs after overexpression ( ${ }^{* *} p<0.05$ compared with the oeNC group); $C$. Cell viability of RBMECs transfected with oeNC and oeFGF21 (** $p<0.05$ compared to the oeNC group); D. BCl-2, Bax and caspase-3 RNA expression in RBMECs transfected with oeNC and oeFGF21 were assessed using RT-qPCR (**p $<0.05$ compared to the oeNC group); E. ELISA was used to detect TNF- $a$ and TGF- $\beta 1$ protein levels in RBMECs after oeNC and oeFGF21 transfection (**p $<0.05$ compared to the oeNC group); F. Phosphorylated ERK1/2 and total ERK1/2 were measured with ELISA in RBMECs after oeNC and oeFGF21 transfection ( ${ }^{* *} p<0.05$ compared to the oeNC group).

IL-1 $\beta$ exhibited a significantly lower level of Bcl-2, and a higher expression of Bax and caspase-3, compared to RBMECs without treatment ( $\mathrm{p}<0.05$, Fig. $1 \mathrm{C}$ ). Inflammatory cytokines were also examined and it was shown that TNF- $\alpha$ was significantly increased, and TGF- $\beta 1$ significantly decreased by treatment with IL- $1 \beta$ ( $\mathrm{p}<0.05$, Fig. 1D). Moreover, the wound healing of RBMECs after Il- $1 \beta$ treatment was examined, and the results indicated that RBMECs after IL- $1 \beta$ treatment had a significantly larger migration area than untreated RBMECs $(\mathrm{p}<0.05$, Fig. 1E). To better understand the functions of inflammation in wound healing, RBMECs treated with IL-1 $\beta$ were further examined.

\section{Overexpression of FGF21 promotes} cell viability, and inhibits cell apoptosis and inflammation of RBMECs via the ERK1/2 signaling pathway

The RT-qPCR showed that FGF21 RNA expression was significantly upregulated after IL-1 $\beta$ treatment $(\mathrm{p}<0.05$, Fig. 2A). Based on this finding, we overexpressed FGF21 in RBMECs to examine the potential functions of this factor in cell growth. FGF21 RNA expression in RBMECs transfected with a compounded fragment of FGF21 was significantly upregulated compared to RBMECs transfected with a NC ( $<<0.05$, Fig. 2B). The CCK- 8 assays showed that overexpressed FGF21 greatly increased the cell viability of RBMECs ( $p<0.05$, Fig. 2 C). In addition, Bcl-2 
was significantly upregulated, and Bax and caspase-3 were markedly downregulated by FGF21 upregulation ( $\mathrm{p}<0.05$, Fig. 2D). Moreover, overexpressed FGF21 suppressed the inflammatory response, which was manifested by a low expression of TNF- $\alpha$ and upregulated TGF- $\beta 1$ ( $\mathrm{p}<0.05$, Fig. 2E). The FGF21 mediated progression of wound healing was also examined, showing that the ERK1/2 signaling pathway was activated through phosphorylated ERK1/2 proteins ( $\mathrm{p}<0.05$, Fig. $2 \mathrm{~F})$.

\section{Upregulated TNF- $a$ inhibits cell viability and promotes the apoptosis of RBMECs through suppressing FGF21}

As TNF- $\alpha$ was downregulated by FGF21 overexpression, the role of this factor in the wound healing of RBMECs was further analyzed. Overexpression of TNF- $\alpha$ significantly increased the protein level of TNF- $\alpha$ compared to the NC group ( $<<0.05$, Fig. 3A). Thereafter, FGF21 RNA expression was measured, indicating that the increased expression of FGF21 caused by the overexpression of FGF21 was reversed to a lower level by TNF- $\alpha$ ( $p<0.05$, Fig. 3B). Furthermore, the increased cell viability of RBMECs after FGF21 upregulation was significantly inhibited by TNF- $\alpha$ promotion, while overexpressed TNF- $\alpha$ increased the apoptosis rate of RBMECs whose apoptosis rate was declined by overexpressed FGF21 ( $\mathrm{p}<0.05$, Fig. 3C,D). Moreover, $\mathrm{Bcl}-2$ expression was decreased, while Bax and caspase- 3 were both promoted after TNF- $\alpha$ overexpression ( $\mathrm{p}<0.05$, Fig. 3E). The scratch test also indicated that overexpressed FGF21 significantly decreased the healing area of RBMECs, while TNF- $\alpha$ upregulation reversed the impact of FGF21 and reduced the migration of RBMECs ( $<<0.05$, Fig. 3F).

\section{Overexpression of VEGFA promotes cell viability and represses apoptosis of RBMECs through the ERK $1 / 2$ signaling pathway}

Because of TNF- $\alpha$ upregulation, FGF21 was significantly downregulated. This treatment also decreased the RNA levels of VEGFA ( $p<0.05$, Fig. 4A). Thus, to examine the functions of VEGFA in RBMEC wound healing, an overexpression of VEGFA was carried out. After upregulation, VEGFA RNA expression was largely increased compared to the NC transfection group ( $\mathrm{p}<0.05$, Fig. $4 \mathrm{~B})$. The cell viability
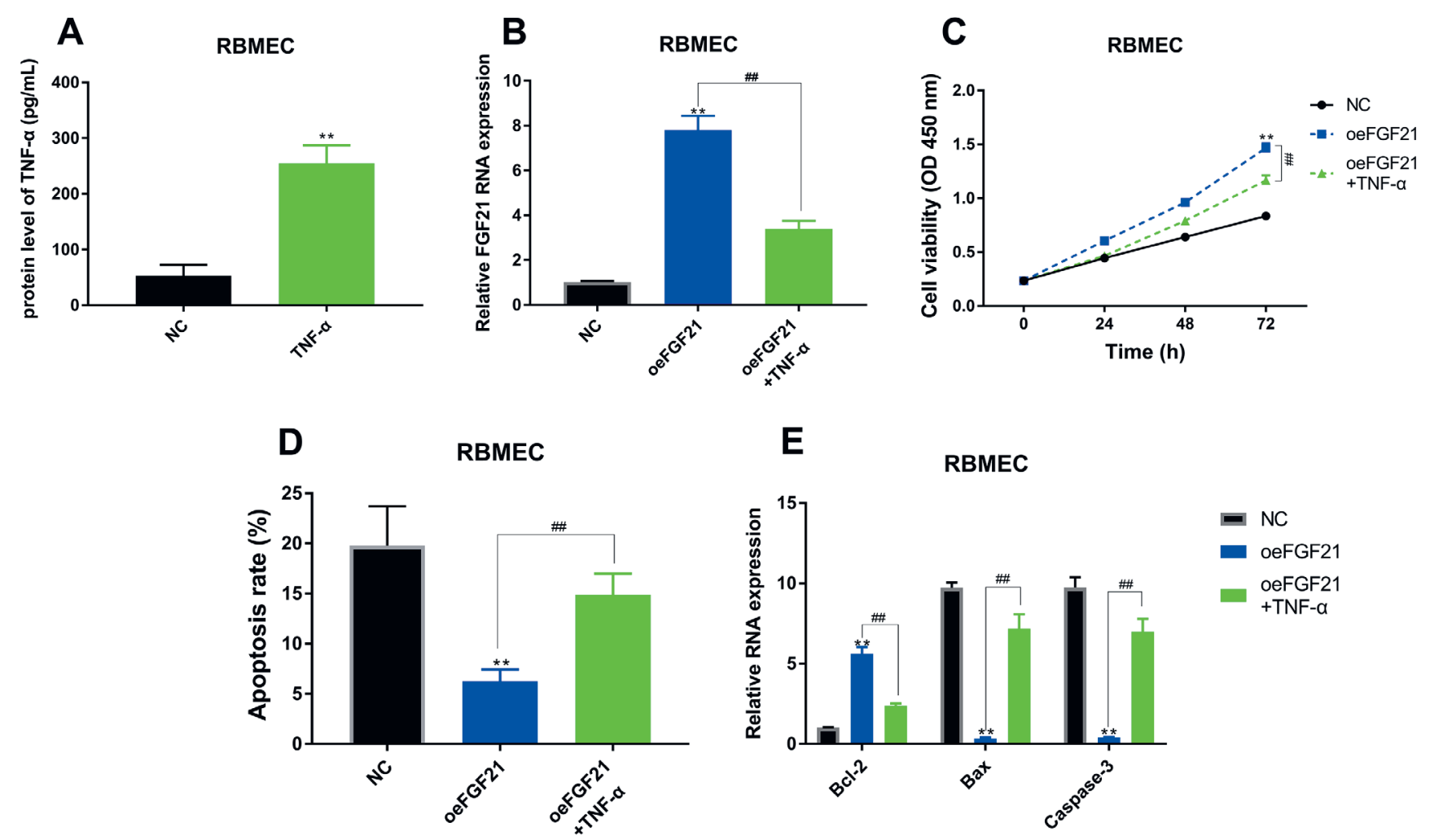

Fig. 3. Upregulated TNF-a inhibits cell viability and promotes cell apoptosis of RBMECs through the suppression of FGF21

A. Protein expression of TNF-a in RBMECs after TNF-a upregulation was evaluated with ELISA (**p $<0.05$ compared to the NC group); B. FGF21 RNA expression after FGF21 overexpression and TNF-a upregulation were detected with RT-qPCR (**p $<0.05$ in comparison with the oeNC group and ${ }^{\# \# p ~}<0.05$ compared to the oeFGF21 group); C. Cell viability of RBMECs after FGF21 and TNF-a overexpression ( ${ }^{* *} p<0.05$ compared to the oeNC group and ${ }^{\# \#} p<0.05$ compared to the oeFGF21 group); D. Flow cytometry was used to measure apoptosis rate of RBMECs after transfection with oeFGF21 and oeTNF-a

${ }^{* *} \mathrm{p}<0.05$ compared to the oeNC group and ${ }^{\# \#} \mathrm{p}<0.05$ compared to the oeFGF21 group); E. RT-qPCR was used to analyze Bcl-2, Bax and caspase-3 RNA expression in RBMECs transfected with FGF21 and TNF-a overexpression (**p $<0.05$ compared to the oeNC group and \#\# $p<0.05$ compared to the oeFGF21 group); F. Scratch test was used to detect wound healing of RBMECs transfected with oeFGF21 and oeTNF-a $*^{* *} p<0.05$ compared to the oeNC group and $\# p<0.05$ compared to the oeFGF21 group). 

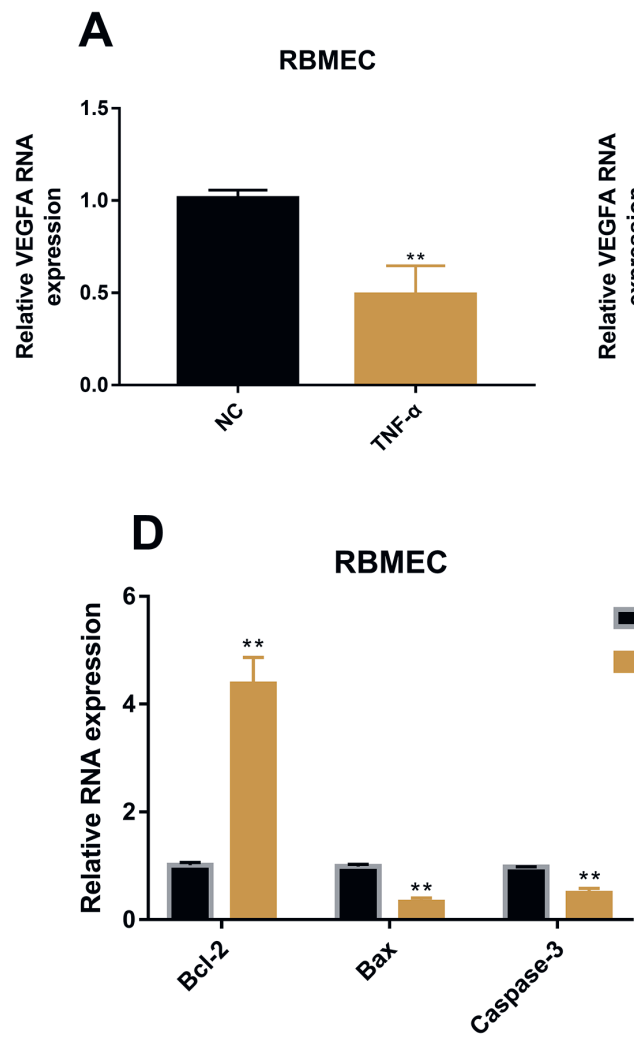

B

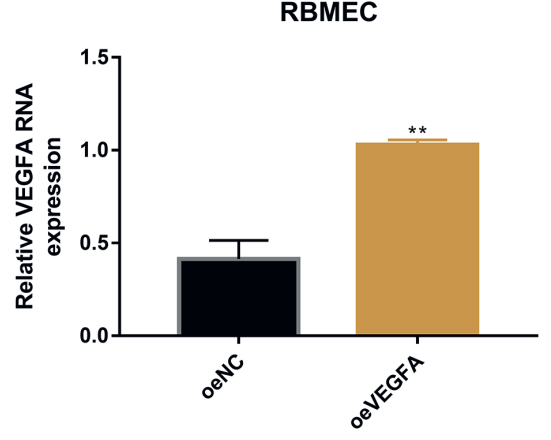

E

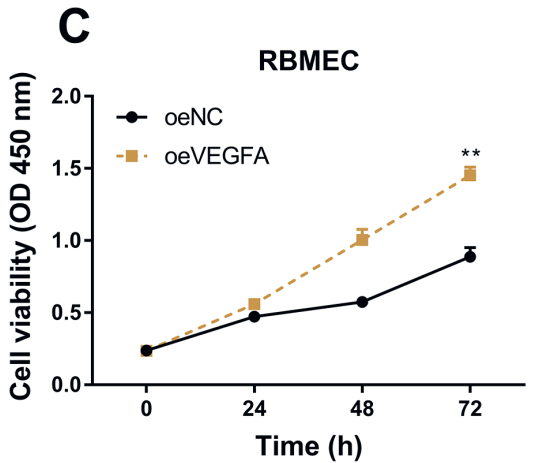

RBMEC

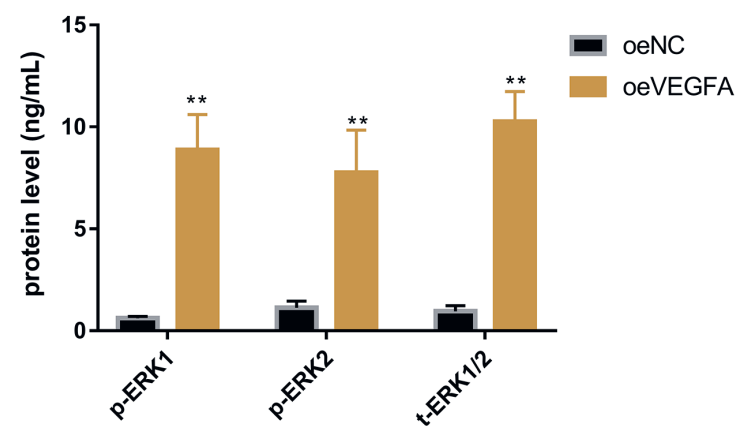

Fig. 4. Overexpression of VEGFA promotes cell viability and represses apoptosis of RBMECs through the ERK1/2 signaling pathway

A. VEGFA RNA expression in normal RBMECs and RBMECs after TNF-a overexpression were assessed with RT-qPCR (**p $<0.05$ compared to the NC group); B. VEGFA RNA expression in RBMECs transfected with oeNC and oeVEGFA were measured with RT-qPCR (** $^{*}<0.05$ compared to the oeNC group); C. CCK-8 was applied to detect cell viability of RBMECs after oeNC and oeVEGFA transfection (**p $<0.05$ compared to the oeNC group); D. BCl-2, Bax and caspase-3 RNA expression were detected with RT-qPCR in RBMECs after oeNC or oeVEGFA transfection (**p $<0.05$ compared to the oeNC group); E. ERK1/2 phosphorylation and total ERK1/2 were measured with ELISA in RBMECs transfected using oeNC and oeVEGFA $\left(^{* *} \mathrm{p}<0.05\right.$ compared to the oeNC group).

of RBMECs was significantly improved by VEGFA overexpression ( $p<0.05$, Fig. $4 \mathrm{C}$ ). In addition, RT-qPCR indicated that the Bcl-2 RNA level was significantly upregulated after VEGFA overexpression, while Bax and caspase-3 RNA expression were both significantly lower compared to the NC group ( $\mathrm{p}<0.05$, Fig. 4D). To investigate the mechanism by which VEGFA mediates cell viability and apoptosis, the effects of this factor on ERK phosphorylation were also examined. The results showed that the protein expression of phosphorylated ERK1/2 was significantly upregulated by VEGFA upregulation ( $<<0.05$, Fig. $4 \mathrm{E})$.

\section{Suppression of the ERK1/2 signaling pathway inhibits the effects of FGF21 and VEGFA on cell viability and apoptosis of RBMECS}

As the ERK1/2 signaling pathway was activated by FGF21 and VEGFA in RBMECs, we examined the potential functions of this pathway in the wound healing progression of these cells. To this end, P98059, an ERK1/2 signaling pathway suppressor, was used to block activation of ERK1/2. PD98059 treatment significantly inhibited not only the protein levels of phosphorylated ERK1/2, but total ERK1/2 protein expression ( $\mathrm{p}<0.05$, Fig. $5 \mathrm{~A}$ ).
Thereafter, the roles of the ERK1/2 signaling pathway were examined after FGF21 and VEGFA were upregulated. PD98059 treatment significantly inhibited the increased cell viability caused by overexpressed FGF21 and VEGFA ( $<<0.05$, Fig. 5B), and enhanced the apoptosis rate of RBMEC after suppression by FGF21 and VEGFA upregulation ( $\mathrm{p}<0.05$, Fig. $5 \mathrm{C}$ ). Moreover, the high levels of Bcl-2 caused by upregulated FGF21 and VEGFA were reversed after PD98059 treatment, while the lower levels of Bax and capase- 3 were both upregulated ( $p<0.05$, Fig. 5D). The scratch test showed that the decreased migration areas induced by overexpressed FGF21 and VEGF were inhibited by PD98059 treatment in RBMECs $(p<0.05$, Fig. 5E).

\section{Discussion}

Endovascular surgery has been shown to treat stroke successfully. ${ }^{28}$ However, the vessel damage that can result from mechanical thrombectomy ${ }^{29}$ is an urgent clinical problem that needs to be addressed. ${ }^{30-32}$ Accelerating the efficiency of wound healing following this procedure might bring a better future for endovascular surgeries. In the progression of wound healing, inflammation is an important step 

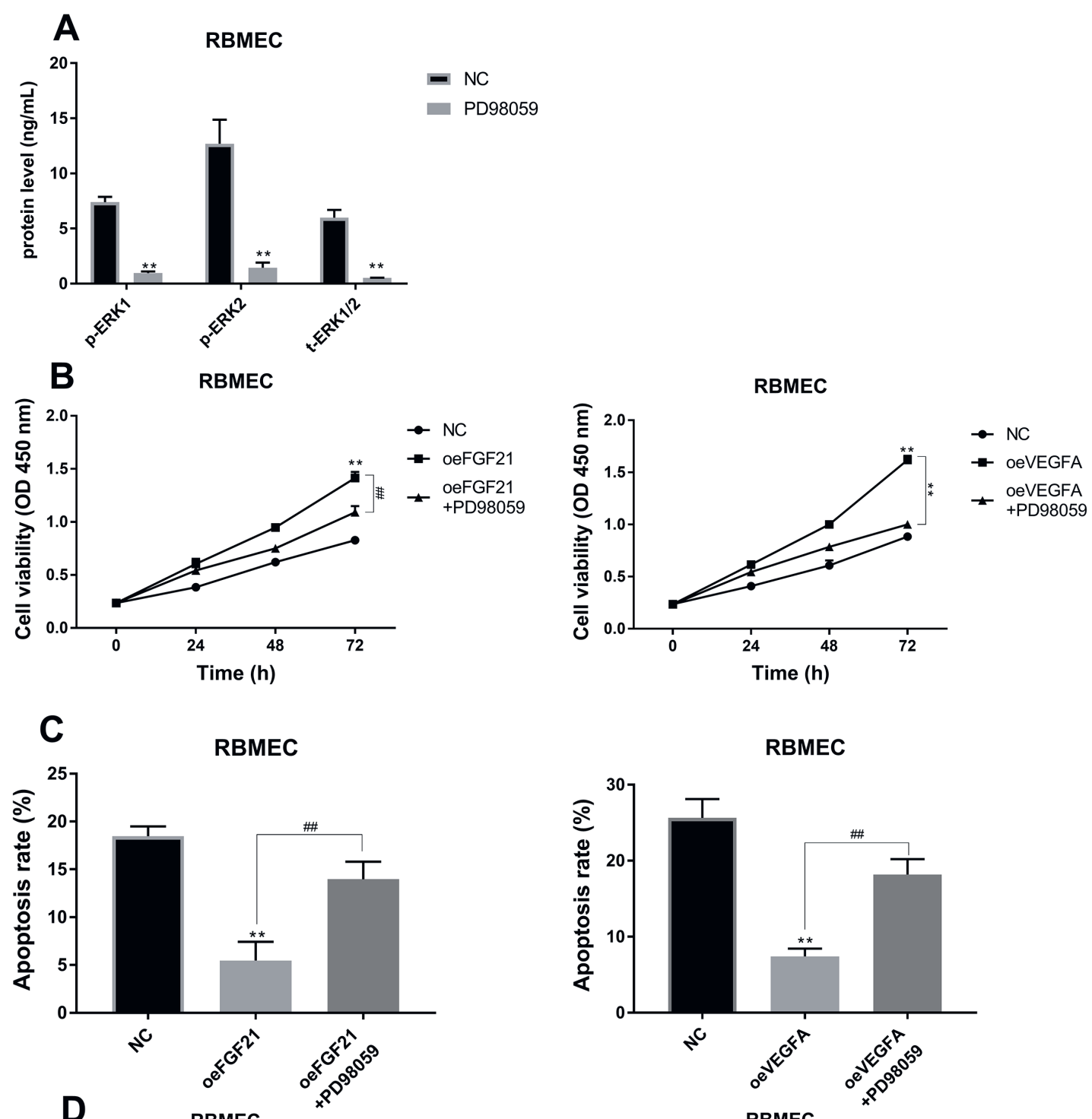

RBMEC
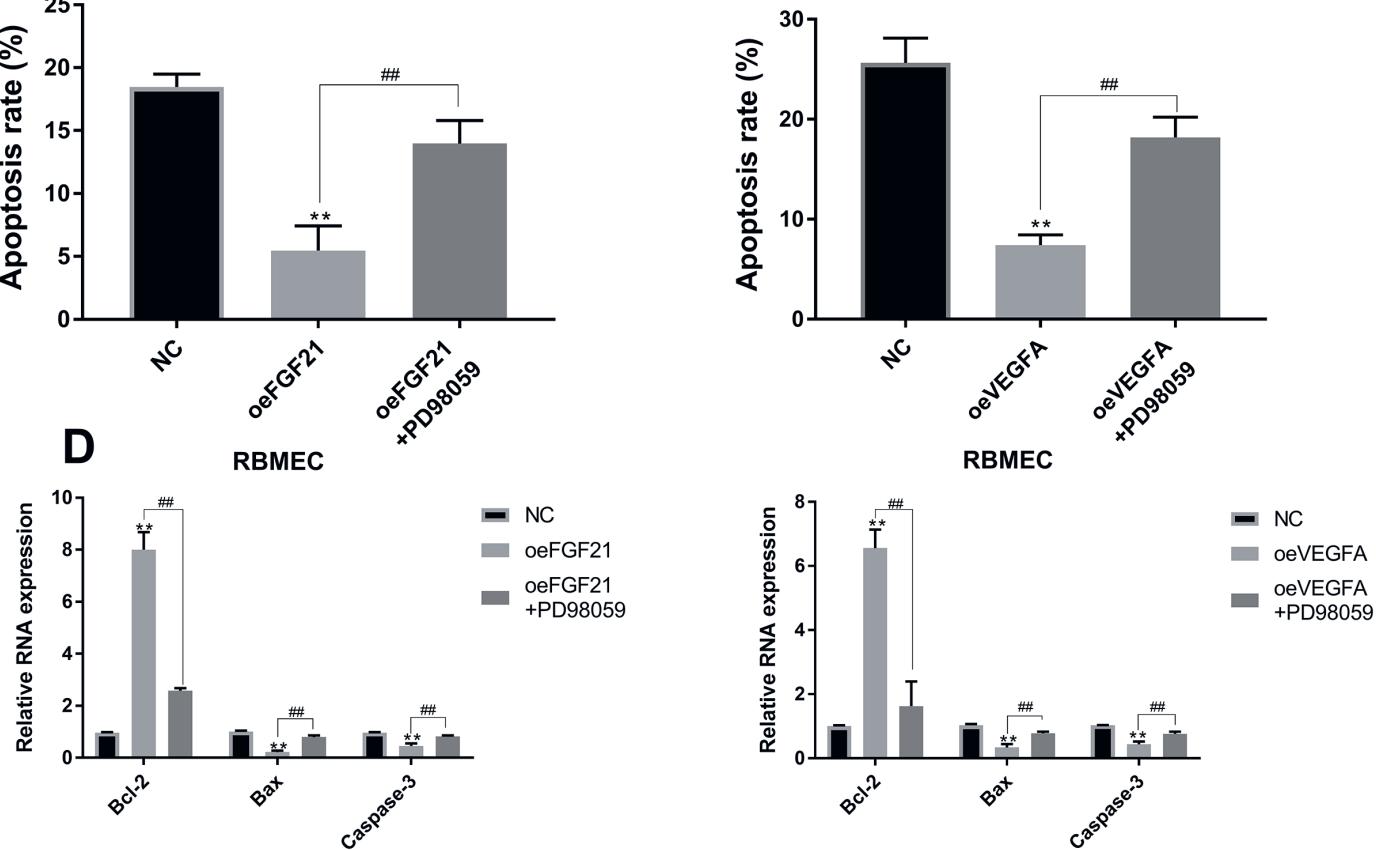

Fig. 5. Suppression of the ERK1/2 signaling pathway inhibits FGF21 and VEGFA regulation of RBMEC viability and apoptosis

A. Phosphorylated ERK1/2 and total ERK1/2 were measured in normal and PD98059 (50 $\mu \mathrm{M})$-treated RBMECs (**p $<0.05$ compared to the NC group); B. Cell viability of RBMEC s after overexpression of FGF21 or VEGFA and overexpression of FGF21 or VEGFA with PD98059 were examined through CCK-8 ${ }^{* *} \mathrm{p}<0.05$ compared to the oeNC group and \#\#p 0.05 compared to the oeFGF21 or oeVEGFA group); C. Apoptosis rate of RBMECs transfected with oeFGF21 or oeVEGFA, and RBEMCs treated with PD98059 after transfection were evaluated using flow cytometry ${ }^{* *} \mathrm{p}<0.05$ compared to the oeNC group and $\# \mathrm{p}<0.05$ compared to the oeFGF21 or oeVEGFA group); D. BCl-2, Bax and caspase-3 RNA expression were measured using RT-qPCR in RBMECs transfected with oeFGF21 and oeVEGFA and PD98059 treatment ${ }^{* *} \mathrm{p}<0.05$ compared to the oeNC group and ${ }^{\# \#} \mathrm{p}<0.05$ compared to the oeFGF21 or oeVEGFA group); E. Wound healing of RBMECs after oeFGF21 and oeVEGFA transfection and PD98059 treatment were checked using the scratch test ${ }^{* *} \mathrm{p}<0.05$ compared to the oeNC group and ${ }^{\# \#} p<0.05$ compared to the oeFGF21 or oeVEGFA group).

that can induce immune system activation, restore homeostasis and repair tissue damage. ${ }^{33}$ However, persistent and overactivated inflammation can result in further damage. ${ }^{34}$ In examinations of inflammatory cytokines, TNF- $\alpha$ has been identified as a factor that can inhibit the metastasis of keratinocytes through upregulation of TIMP-1 expression, but a TNF- $\alpha$ antagonist has been shown to improve the progression of wound healing in diabetic rats models. ${ }^{35}$ 
By contrast, TGF- $\beta 1$ has also been reported to attenuate the inflammatory response via the ERK1/2 signaling pathway and can facilitate the progression of wound healing. ${ }^{36,37}$ Based on these studies, we examined the effects of inflammation on the wound healing of RBMECs. Using CCK-8 assays and flow cytometry, we determined that RBMECs treated with IL-1 $\beta$ showed lower cell viability and a higher cell apoptosis rate. Moreover, downregulated Bcl-2 RNA expression, and upregulated Bax and caspase- 3 expression, also indicated that IL-1 $\beta$ facilitated cell apoptosis. As for the effects of IL-1 $\beta$ on inflammatory cytokines, TNF- $\alpha$ protein expression was upregulated, while TGF- $\beta 1$ was significantly decreased. Hence, in the current study, prolonged inflammation in RBMECs has been shown to decrease cell viability and migration, and to promote cell apoptosis, through the stimulation of TNF- $\alpha$ and the suppression of TGF- $\beta 1$.

Studies examining the functions of FGF21 have commonly focused on homeostasis, especially its role in energy metabolism. ${ }^{38}$ However, FGF21 can also act as an antiinflammatory cytokine, which has been shown in experimental pancreatitis and myocardial ischemia. ${ }^{38}$ In addition, FGF21 has been reported to promote wound healing through increasing the activation of c-Jun $\mathrm{N}$-terminal kinase (JNK). ${ }^{39}$ In rat myocardial ischemia reperfusion and H9c2 hypoxia re-oxygenation models, FGF21 significantly reduced cell apoptosis and inhibited TNF- $\alpha$ through binding miR-145..$^{40}$ However, whether FGF21 can also influence TNF- $\alpha$ during the progression of wound healing has seldom been mentioned. Thus, we have investigated the role of FGF21 in the wound healing of RBMECs, which revealed that FGF21 promoted cell viability and inhibited cell apoptosis. Moreover, FGF21 suppressed the protein levels of TNF- $\alpha$ and increased TGF- $\beta 1$. Therefore, we hypothesized that FGF21 might promote wound healing through a suppression of TNF- $\alpha$. Apart from inflammation, the ERK1/2 signaling pathway has also been shown to play an important role in FGF signaling transmission. ${ }^{38}$ Activation of the ERK1/2 signaling pathway helps FGF21 facilitate glucose uptake by inducing expression of glucose transporter-1 in adipocytes. ${ }^{41}$ Moreover, p-ERK1/2 was activated and promoted during the wound healing of diabetic rats after propranolol treatment. ${ }^{42}$ Based on these studies, we analyzed the expression of ERK1 and ERK2 and showed that these proteins were significantly upregulated. Therefore, FGF21 might accelerate the wound healing of RBMECs through activation of the ERK1/2 signaling pathway.

As outlined above, FGF21 inhibited TNF- $\alpha$ in the RBMEC model. Others have reported that TNF- $\alpha$ can suppress $\beta$-Klotho expression and attenuate the roles of FGF21 in adipocytes. ${ }^{43}$ In the current study, TNF- $\alpha$ also increased cell apoptosis, and reduced the viability and wound healing of RBMECs by inhibiting the functions of FGF21. Therefore, inflammation might retard the progression of wound healing by inhibiting FGF21. Moreover, TNF- $\alpha$ was found to inhibit VEGFA in RBMEC cells, which is an important factor in the progression of wound healing. ${ }^{44}$ The VEGFA has been reported to accelerate angiogenesis in human umbilical vein endothelial cells (HUVECs) via ERK1/2 phosphorylation and ACE2 inhibition. ${ }^{45}$ Therefore, we examined the functions of VEGFA in RBMECs, showing that it could improve cell viability and depress cell apoptosis. Moreover, VEGFA enhanced phosphorylation of ERK1/2 in RBMECs. Therefore, we hypothesized that TNF- $\alpha$ might also inhibit wound healing through downregulating VEGFA.

In the current study, the ERK1/2 signaling pathway was activated by FGF21 and VEGFA. Based on this finding, we speculated if activation of the ERK1/2 signaling pathway was a necessary step in wound healing. To test this idea, we used PD98059 to inhibit ERK1/2. Treatment with this agent significantly repressed the promotion of cell viability and migration caused by FGF21 and VEGFA, while inhibition of the apoptosis rate was also reversed to a higher level. PD98059 has been shown to inhibit the wound healing progression of human keratinocytes by suppressing p-ERK1 and p-ERK2, which was also shown in a rat wound model. ${ }^{46}$ In our study, FGF21 mediated cell viability and cell apoptosis of RBMECs via the inhibition of TNF- $\alpha$ and activation of the ERK1/2 signaling pathway. Furthermore, TNF- $\alpha$ suppressed FGF21 functions and VEGFA, while VEGFA also regulated the viability and apoptosis of RBMECs via the ERK1/2 signaling pathway. Thus, FGF21 may accelerate wound healing through upregulating VEGFA and activating the ERK1/2 signaling pathway by suppressing TNF- $\alpha$. However, this finding will need to be confirmed in future work.

\section{Conclusions}

The FGF21 facilitated the viability and inhibited the apoptosis of RBMECs through the activation of ERK1/2 and VEGFA, caused by inhibition of TNF- $\alpha$. These findings suggest that FGF21 might be a useful factor to improve wound healing. However, in vivo and clinical studies will be needed to demonstrate the effectiveness of this approach.

\section{ORCID iDs}

Weiting Chen (D) https://orcid.org/0000-0001-7624-0754 Zhongen Shen (1) https://orcid.org/0000-0003-4146-1113 Shuiqi Cai (D) https://orcid.org/0000-0002-1983-1029 Long Chen (D) https://orcid.org/0000-0001-9430-6817 Dabin Wang (D) https://orcid.org/0000-0001-9377-5830

\section{References}

1. Morotti A, Poli P, Costa P. Acute stroke. Semin Neurol. 2019;39(1):61-72. doi:10.1055/s-0038-1676992

2. O'Donnell MJ, Lim Chin S, Rangarajan S, et al; INTERSTROKE investigators. Global and regional effects of potentially modifiable risk factors associated with acute stroke in 32 countries (INTERSTROKE): A case-control study. Lancet. 2016;388(10046):761-775. doi:10.1016/ S0140-6736(16)30506-2

3. Khandelwal P, Yavagal DR, Sacco RL. Acute ischemic stroke intervention. J Am Coll Cardiol. 2016;67(22):2631-2644. doi:10.1016/j.jacc. 2016.03.555

4. Saver JL. Time is brain-quantified. Stroke. 2006;37(1):263-266. doi:10. 1161/01.STR.0000196957.55928.ab 
5. Wang A, Abramowicz AE. Endovascular thrombectomy in acute ischemic stroke: New treatment guide. Curr Opin Anaesthesiol. 2018;31(4): 473-480. doi:10.1097/ACO.0000000000000621

6. Peschillo S, Diana F, Berge J, Missori P. A comparison of acute vascular damage caused by ADAPT versus a stent retriever device after thrombectomy in acute ischemic stroke: A histological and ultrastructural study in an animal model. J Neurointerv Surg. 2017;9(8):743-749. doi:10.1136/neurintsurg-2016-012533

7. Reinke JM, Sorg H. Wound repair and regeneration. Eur Surg Res. 2012;49(1):35-43. doi:10.1159/000339613

8. Mustoe TA, O'Shaughnessy K, Kloeters O. Chronic wound pathogenesis and current treatment strategies: A unifying hypothesis. Plast Reconstr Surg. 2006;117(7 Suppl):35S-41S. doi:10.1097/01.prs.0000225431.63010.1b

9. Ornitz DM, Itoh $\mathrm{N}$. The fibroblast growth factor signaling pathway. Wiley Interdiscip Rev Dev Biol. 2015;4(3):215-266. doi:10.1002/wdev.176

10. Potthoff MJ, Kliewer SA, Mangelsdorf DJ. Endocrine fibroblast growth factors 15/19 and 21: From feast to famine. Genes Dev. 2012;26(4):312-324. doi:10.1101/gad.184788.111

11. Salminen A, Kaarniranta K, Kauppinen A. Regulation of longevity by FGF21: Interaction between energy metabolism and stress responses. Ageing Res Rev. 2017;37:79-93. doi:10.1016/j.arr.2017.05.004

12. Fisher FM, Chui PC, Nasser IA, et al. Fibroblast growth factor $21 \mathrm{lim}$ its lipotoxicity by promoting hepatic fatty acid activation in mice on methionine and choline-deficient diets. Gastroenterology. 2014; 147(5):1073-1083.e6. doi:10.1053/j.gastro.2014.07.044

13. Wang N, Li JY, Li S, et al. Fibroblast growth factor 21 regulates foam cells formation and inflammatory response in Ox-LDL-induced THP-1 macrophages. Biomed Pharmacother. 2018;108:1825-1834. doi:10. 1016/j.biopha.2018.09.143

14. Liu H, Zhao Y, Zou Y, et al. Heparin-poloxamer hydrogel-encapsulated rhFGF21 enhances wound healing in diabetic mice. FASEB J. 2019;33(9):9858-9870. doi:10.1096/fj.201802600RR

15. Coskun T, Bina HA, Schneider MA, et al. Fibroblast growth factor 21 corrects obesity in mice. Endocrinology. 2008;149(12):6018-6027. doi:10. 1210/en.2008-0816

16. Ye L, Wang X, Cai C, et al. FGF21 promotes functional recovery after hypoxic-ischemic brain injury in neonatal rats by activating the PI3K/Akt signaling pathway via FGFR1/ $\beta$-klotho. Exp Neurol. 2019;317:34-50. doi:10.1016/j.expneurol.2019.02.013

17. Nissen NN, Polverini PJ, Koch AE, Volin MV, Gamelli RL, DiPietro LA. Vascular endothelial growth factor mediates angiogenic activity during the proliferative phase of wound healing. Am J Pathol. 1998;152(6): 1445-1452. PMID:9626049

18. Lim JC, Mattos M, Fang M, et al. TNFa contributes to diabetes impaired angiogenesis in fracture healing. Bone. 2017;99:26-38. doi:10.1016/j. bone.2017.02.014

19. Jeon $\mathrm{HH}, \mathrm{Yu} \mathrm{Q}, \mathrm{Lu} \mathrm{Y}$, et al. FOXO1 regulates VEGFA expression and promotes angiogenesis in healing wounds. J Pathol. 2018;245(3): 258-264. doi:10.1002/path.5075

20. Chamorro-Jorganes A, Lee MY, Araldi E, et al. VEGF-induced expression of miR-17-92 cluster in endothelial cells is mediated by ERK/ELK1 activation and regulates angiogenesis. Circ Res. 2016;118(1):38-47. doi:10.1161/CIRCRESAHA.115.307408

21. Lee BC, Song J, Lee A, Cho D, Kim TS. Visfatin promotes wound healing through the activation of ERK1/2 and JNK1/2 pathway. Int J Mol Sci. 2018;19(11):3642. doi:10.3390/ijms19113642

22. Wang $R$, et al. FGF21 regulates melanogenesis in alpaca melanocytes via ERK1/2-mediated MITF downregulation. Biochem Biophys Res Commun. 2017;490(2):466-471. doi:10.1016/j.bbrc.2017.06.064

23. Liu Q, Wang S, Wei M, et al. Improved FGF21 sensitivity and restored FGF21 signaling pathway in high-fat diet/streptozotocin-induced diabetic rats after duodenal-jejunal bypass and sleeve gastrectomy. Front Endocrinol (Lausanne). 2019;10:566. doi:10.3389/fendo.2019.00566

24. Cui J, Gong C, Cao B, Li L. MicroRNA-27a participates in the pathological process of depression in rats by regulating VEGFA. Exp TherMed. 2018;15(5):4349-4355. doi:10.3892/etm.2018.5942

25. Mahdavi S, Khodarahmi P, Roodbari NH. Effects of cadmium on Bcl-2/ Bax expression ratio in rat cortex brain and hippocampus. Hum Exp Toxicol. 2018;37(3):321-328. doi:10.1177/0960327117703687

26. Yin HY, Wei JR, Zhang R, Ye XL, Zhu YF, Li WJ. Effect of glutamine on caspase- 3 mRNA and protein expression in the myocardium of rats with sepsis. Am J Med Sci. 2014;348(4):315-318. doi:10.1097/MAJ.000 0000000000237
27. Martuscello RT, Spengler RM, Boniu AC, et al. Increasing TNF levels solely in the rat hippocampus produces persistent pain-like symptoms. Pain. 2012;153(9):1871-1882. doi:10.1016/j.pain.2012.05.028

28. Goyal M, Menon BK, van Zwam WH, et al; HERMES collaborators. Endovascular thrombectomy after large-vessel ischaemic stroke: A metaanalysis of individual patient data from five randomised trials. Lancet. 2016;387(10029):1723-1731. doi:10.1016/S0140-6736(16)00163-X

29. Koge J, Kato S, Hashimoto T, Nakamura Y, Kawajiri M, Yamada T. Vessel wall injury after stent retriever thrombectomy for internal carotid artery occlusion with duplicated middle cerebral artery. World Neurosurg. 2019;123:54-58. doi:10.1016/j.wneu.2018.11.223

30. Leishangthem L, Satti SR. Vessel perforation during withdrawal of Trevo ProVue stent retriever during mechanical thrombectomy for acute ischemic stroke. J Neurosurg. 2014;121(4):995-998. doi:10.3171/2014. 4.JNS132187

31. Arai $D$, Ishii A, Chihara $H$, Ikeda $H$, Miyamoto $S$. Histological examination of vascular damage caused by stent retriever thrombectomy devices. J Neurointerv Surg. 2016;8(10):992-995. doi:10.1136/neur intsurg-2015-011968

32. Truong M, Bloch KM, Andersen M, Andsberg G, Töger J, Wassélius J. Subacute vessel wall imaging at 7-TMRI in post-thrombectomy stroke patients. Neuroradiology. 2019;61(10):1145-1153. doi:10.1007/s00234019-02242-9

33. Landén NX, Li D, Ståhle M. Transition from inflammation to proliferation: A critical step during wound healing. Cell Mol Life Sci. 2016;73(20): 3861-3885. doi:10.1007/s00018-016-2268-0

34. Smigiel KS, Parks WC. Macrophages, wound healing, and fibrosis: Recent insights. Curr Rheumatol Rep. 2018;20(4):17. doi:10.1007/s11926018-0725-5

35. Huang SM, Wu CS, Chiu MH, et al. High glucose environment induces M1 macrophage polarization that impairs keratinocyte migration via TNF-a: An important mechanism to delay the diabetic wound healing. J Dermatol Sci. 2019;96(3):159-167. doi:10.1016/j.jdermsci. 2019.11.004

36. Lichtman MK, Otero-Vinas M, Falanga V. Transforming growth factor beta (TGF- $\beta$ ) isoforms in wound healing and fibrosis. Wound Repair Regen. 2016;24(2):215-222. doi:10.1111/wrr.12398

37. Zhang J, Li Z, Chen F, et al. TGF- $\beta 1$ suppresses CCL3/4 expression through the ERK signaling pathway and inhibits intervertebral disc degeneration and inflammation-related pain in a rat model. Exp Mol Med. 2017;49(9):e379. doi:10.1038/emm.2017.136

38. Fisher FM, Maratos-Flier E. Understanding the physiology of FGF21. Annu Rev Physiol. 2016;78:223-241. doi:10.1146/annurev-physiol021115-105339

39. Song YH, Zhu YT, Ding J, et al. Distribution of fibroblast growth factors and their roles in skin fibroblast cell migration. Mol Med Rep. 2016;14(4):3336-3342. doi:10.3892/mmr.2016.5646

40. Hu S, Cao S, Tong Z, Liu J. FGF21 protects myocardial ischemia-reperfusion injury through reduction of miR-145-mediated autophagy. Am J Transl Res. 2018;10(11):3677-3688. PMID:30662618

41. Ge X, Chen C, Hui X, Wang Y, Lam KS, Xu A. Fibroblast growth factor 21 induces glucose transporter-1 expression through activation of the serum response factor/Ets-like protein-1 in adipocytes. J Biol Chem. 2011;286(40):34533-34541. doi:10.1074/jbc.M111.248591

42. Chang X, Li S, Xue XD, Chang F. Propranolol regulates ERK1/2 signaling pathway and promotes chronic wound healing in diabetic rats. Eur Rev Med Pharmacol Sci. 2019;23(10):4498-4506. doi:10.26355/ eurrev_201905_17962

43. Díaz-Delfín J, Hondares E, Iglesias R, Giralt M, Caelles C, Villarroya F. TNF-a represses $\beta$-Klotho expression and impairs FGF21 action in adipose cells: Involvement of JNK1 in the FGF21 pathway. Endocrinology. 2012;153(9):4238-4245. doi:10.1210/en.2012-1193

44. Ren S, Chen J, Duscher D, et al. Microvesicles from human adipose stem cells promote wound healing by optimizing cellular functions via AKT and ERK signaling pathways. Stem Cell Res Ther. 2019;10(1):47. doi:10.1186/s13287-019-1152-x

45. Zhang Q, Lu S, Li T, et al. ACE2 inhibits breast cancer angiogenesis via suppressing the VEGFa/VEGFR2/ERK pathway. J Exp Clin Cancer Res. 2019;38(1):173. doi:10.1186/s13046-019-1156-5

46. Wang Y, Zheng J, Han Y, et al. JAM-A knockdown accelerates the proliferation and migration of human keratinocytes, and improves wound healing in rats via FAK/Erk signaling. Cell Death Dis. 2018;9(9):848. doi:10.1038/s41419-018-0941-y 